\title{
Effect of Sowing and Nitrogen Application Methods on Yield and Yield Components of Some Wheat Varieties
}

\author{
S. A. S. Mehasen *; N. Kh. B. El-Gizawy; M. E. R. Gomaa and A. M. A. Halawa \\ Agron. Dep., Fac. of Agric., Moshtohor, Benha Univ., Egypt. \\ *Corresponding author: Sadiek Abdelaziz Sadiek Mehasen, Head Department of Agronomy, Faculty of \\ Agriculture at moshtohor, Benha University, Egypt. PO Box 13736.
}

\begin{abstract}
Two field experiments were conducted at the Experiment and Research Center, Fac. Agric., Moshtohor, Benha Univ., during 2014/15 and 2015/16 seasons. The aim of this study was response to investigate the effect of three wheat varieties (Misr 2, Sakha 94 , and Sids 12)to four planting methods (prodcasting, rows, hills on ridges and rows on beds ) and two application methods (prodcasting and drilling).

The results indicated that:

Misr 2 variety gave the highest values of plant height, spike length, No. tillers and spikes $\mathrm{m}^{-2}$, No. spikelets spike $^{-1}$, weight of grains spike ${ }^{-1}$, biological and grain yields fed ${ }^{-1}$ compared with the other varieties in the first and second seasons. planting by rows on bed method increased significantly plant height, spike length, No. otillers and spikes $\mathrm{m}^{-2}$, No. spikelets spike ${ }^{-1}$, weight of grains spike ${ }^{-1}, 1000$-grain weight, biological and grain yields fed ${ }^{-1}$ compared with the other planting methods in the first and second seasons.

Plant height, spike length, No. tillers and spikes $\mathrm{m}^{-2}$, No. spikelets spike ${ }^{-1}$, weight of grains spike ${ }^{-1}, 1000$ grain weight, biological and grain yields fed $^{-1}$ increased significantly by prodcasting $\mathrm{N}$ application method in the first and second seasons.

Plant height, No. tillers and spikes $\mathrm{m}^{-2}$, No. spikelets spike ${ }^{-1}$, weight of grains spike ${ }^{-1}, 1000$-grain weight, biological and grain yields fed ${ }^{-1}$ were affected by the interaction between wheat varieties and planting methods in the first and second seasons except 1000- grain weight in the second season.

Plant height, No. tillers and spikes $\mathrm{m}^{-2}$, No. spikelets spike ${ }^{-1}, 1000$ - grain weight and biological yield fed ${ }^{-1}$ were affected by the interaction between varieties and nitrogen application methods in the first and second seasons except plant height and No. spikelets spike ${ }^{-1}$ in the first season. Plant height, No. tillers and spikes $\mathrm{m}^{-2}$, No. spikelets spike ${ }^{-1}$, biological and grain yields $f^{-1}{ }^{-1}$ were affected by the interaction between planting methods and nitrogen application methods in the first and second seasons except plant height, No. tillers $\mathrm{m}^{-2}$, No. spikelets spike ${ }^{-1}$ and grain yield fed ${ }^{-1}$ in the first season. Also, plant height, No. tillers and spikes $\mathrm{m}^{-2}$, weight of grains spike ${ }^{-1}$, 1000-grain weight, biological and grain yields $\mathrm{fed}^{-1}$ responded significant to the interaction of the three involved factors (varieties x planting methods $\mathrm{x} N$ application methods).

It could be concluded that under the conditions of the experiment, planting Misr 2 var by rows on bed method under proadcasting $\mathrm{N}$ application is recommended.
\end{abstract}

Key Words: Spring wheat varieties, planting methods, $\mathrm{N}$ application methods, Yield and its components.

\section{Introduction}

Wheat is considered the main source of food in the world and in Egypt. Raising wheat production through increasing productivity and increasing the cultivated area is an important national target to minimize the gap between the Egyptian production and consumption. The total production of wheat reached at least 9,000,000 tons annually (FAO, 2016). Increasing wheat yield per unit area can be achieved by breeding high yielding varieties or improving the cultural treatments of the crop. Modern wheat varieties were developed to maximize grain yield under favorable environmental conditions (high input conditions especially planting methods to save water supply and seeding rate). In the light of the present national water policy concerning saving irrigation water expanding wheat area needs.

Several investigators showed that wheat cultivars differed in yield and its components as well as chemical properties (Mehasen, 1999; El-Hawary, 2000; Abd El-hameed, 2002; Ali etal, 2004; Mehasen and Mohamed, 2005; Abu-Grab etal, 2006; Omar, 2007; Hassan, 2008; Mehasen etal, 2009; Ashmawy etal, 2010; Abd El-Nour and Fateh, 2011; Mehasen etal, 2013; Mehasen etal, 2014 ; Mehasen etal, 2015).( Zenhom etal, (2018) reported that wheat cultivars were varied significantly in plant height, No. tillers $\mathrm{m}^{-2}$, No. spikes $\mathrm{m}^{-2}$, seed index and grain yield fed $^{-1}$.

Wheat is planted with different sowing methods depending upon the available soil water, time of planting, amount of preceding crop residues in the field and availability of planting machinery (Sikander etal., 2003). The method of sowing is significant as it determines the proper crop stand establishment and the production of individual plant depends on balancing plant to plant competition (Sulieman, 2010). Results revealed that all yield and yield parameters were significantly affected by the 
sowing method (Abd El-Lattief, 2011). Generally, planting on ridge and ridge with line achieved higher 1000 -seed weight and grain yield for both seasons (Dagash etal, 2014). Better plant height was noted in drill planting with $17.5,20 \mathrm{~cm}$ rows and $15: 25 \mathrm{~cm}$ paired rows. However, number of spikelets spike ${ }^{-1}$ and number of grains spike ${ }^{-1}$ were statistically similar in drilling at $17.5,20$, and $15: 25 \mathrm{~cm}$ paired apart rows. Similarly, 1000 grain weight was recorded in drill sowing at $20 \mathrm{~cm}$ and $15: 25 \mathrm{~cm}$ paired rows. The maximum grain yield was obtained through $15: 25 \mathrm{~cm}$ paired rows drill planting method and it was statistically at par with drill planting method, where ,row spacing was $20 \mathrm{~cm}$. Whereas, drill-planting techniques with row spacing $15 \mathrm{~cm}$ was inferior to broadcast method (Naresh etal, 2014). The maximum grain yield of $3.5 \mathrm{t} \mathrm{ha}^{-1}$ was obtained from row planting and the least $3.13 \mathrm{t} \mathrm{ha}^{-1}$ was recorded from broadcast method (Tadesse etal, 2017).

Appropriate fertilizer application methods enhance the nutrient use efficiency by reducing their losses. Significant effect of increased $\mathrm{N}$ applied as side dressing on the number of wheat yield per unit area has been reported by Teixeira- Filho etal (2007). Mohammed et al, (2013) reported that significantly higher grain yield $(32.5 \mathrm{bu} / \mathrm{ac})$ was recorded from the fall application of ESN than broadcast (RUBF) and with seed (RUSF). However, (Hassan etal ,2008) reported that nitrogen broadcast or side-dressing even at higher nitrogen rates $(180 \mathrm{~kg} / \mathrm{ha})$ did not produce significantly higher values of crop growth parameters. Ullah etal (2013) reported that Days taken to heading showed non-significant effect of $\mathrm{N}$ fertilizer application methods (broadcast ,sidedressing) in both (seasons) .

The present investigation aimed to study the effect of four planting methods and two nitrogen application methods on yield and its components for three wheat varieties grown on a clay soil in Kalubia Governorate.

\section{Materials and Methods}

Two field experiments were carried out in the Experimental Field of the Faculty of Agriculture at Mashtohor, Benha Univ., during 2014/15 and $2015 / 16$ seasons, to study the effect of three wheat varieties (Misr 2, Sakha 94, Sids 12), four different planting methods (prodcasting, rows, hills on ridges and rows on beds ) and two application methods of nitrogen fertilizer (prodcasting, drilling) on yield and yield components. The soil was clay in texture with a PH value of 7.80 and 7.82 and an organic matter content of 1.82 and $1.83 \%$ and available $\mathrm{N}$ of 53 and $54 \mathrm{ppm}$ during the first and second seasons. Respectively.

Every experiment included twenty four treatments which were the combination of three wheat varieties, four planting methods and two nitrogen applying methods. The experimental design was a split split-plot with four replications. The three wheat varieties were arranged at random in the main plots, the sub- plots were assigned random by to the four different randomly planting methods and the two application methods of nitrogen were arranged random by in the sub sub-plots. The sub sub-plot area was $10.5 \mathrm{~m}^{2}(3 \times 3.5 \mathrm{~m})$. Wheat varieties were planted in November $19^{\text {th }}$ and $18^{\text {th }}$ in the first and second seasons. In the two seasons, the preceding crop was carrot. The normal cultural practices for growing wheat were followed as recommended for the region.

Random samples of 10 guarded plants were taken from sub sub-plots at harvesting time to determine the following characters: plant height $(\mathrm{cm})$, spike length $(\mathrm{cm})$, number of spikelets spike ${ }^{-1}$ and weight of grains $\operatorname{spike}^{-1}(\mathrm{~g})$. For determining No. tillers and spikes $\mathrm{m}^{-2}$ and 1000-grain weight (g) a sample of one square meter from each sub sub-plot was taken. Grain and straw yields $\left(\mathrm{kg} \mathrm{fed}^{-1}\right)$ were estimated on whole sub sub-plot basis.

Analysis of variance was done for the data of each season separately according to Snedecor and Cochran (1980). Treatment means were compared using least significant difference test at 0.05 level of significance, Using the MSTAT-C Statistical Software package (Michigan State University, 1983)

\section{Results and Discussion}

\section{Varietal differences}

The results reported in Table (1) indicate clearly that, there were significant differences among wheat varieties in all studied traits in the first and second seasons. Misr 2 variety gave the highest values of plant height, spike length, No. tillers and spikes $\mathrm{m}^{-2}$, number of spikelets spike ${ }^{-1}$, weight of grains spike ${ }^{-1}$, biological and grain yields fed $^{-1}$ compared with the other varieties in the first and second seasons. Whereas, Sakha 94 variety gave the highest value and increased significantly 1000-grain weight compared with the other varieties in the first and second seasons.

It could be concluded that varietal differences among wheat varieties may be due to genetical make up. The superiority of Misr 2 variety in grain yield fed $^{-1}$ over other varieties might be due to the increase in yield components, namely, spike length, No.tillers and spikes $\mathrm{m}^{-2}$, number of spikelets spike ${ }^{-1}$, weight of grains spike ${ }^{-1}$. The results obtained by Mehasen (1999); El-Hawary (2000); Abd El-hameed (2002); Ali etal, (2004); Mehasen and Mohamed (2005); Abu-Grab et al, (2006); Omar (2007); Hassan (2008); Mehasen et al, (2009); Ashmawy etal, (2010); Abd El-Nour and Fateh (2011); Mehasen etal, (2013); Mehasen etal, (2014); Mehasen etal, (2015); Abo-Remaila and Abou El-Enim (2017) and Zenhom etal, (2018) indicated marked differences among wheat varieties in yield and yield components 
Table 1. Varietal differences in yield and yield components at 2014/15 and2015/16 seasons.

\begin{tabular}{|c|c|c|c|c|c|c|c|c|}
\hline \multirow[b]{2}{*}{ Characters } & \multicolumn{3}{|c|}{ 2014/15 season } & \multirow{2}{*}{$\begin{array}{c}\text { LSD } \\
\text { at } \\
\mathbf{5 \%} \\
\end{array}$} & \multicolumn{3}{|c|}{ 2015/16 season } & \multirow{2}{*}{$\begin{array}{c}\text { LSD } \\
\text { at } \\
\mathbf{5 \%} \\
\end{array}$} \\
\hline & $\begin{array}{c}\text { Misr } \\
2\end{array}$ & $\begin{array}{c}\text { Sakha } \\
94\end{array}$ & $\begin{array}{c}\text { Sids } \\
12\end{array}$ & & $\begin{array}{c}\text { Misr } \\
2\end{array}$ & $\begin{array}{c}\text { Sakha } \\
94\end{array}$ & $\begin{array}{c}\text { Sids } \\
12 \\
\end{array}$ & \\
\hline Plant height (cm) & 114.1 & 107.0 & 110.0 & 0.36 & 114.2 & 107.0 & 110.21 & 0.15 \\
\hline Spike length (cm) & 10.62 & 9.50 & 9.75 & 0.16 & 10.71 & 9.62 & 9.83 & 0.19 \\
\hline Number of tillers $\mathbf{~ m}^{-2}$ & 248.0 & 184.33 & 207.12 & 2.37 & 248.8 & 185.2 & 208.1 & 1.91 \\
\hline Number of spikes $\mathbf{m}^{-2}$ & 245.5 & 182.04 & 204.33 & 2.4 & 246.1 & 182.9 & 205.8 & 1.2 \\
\hline Number of spikelets spike & 23.00 & 21.92 & 22.54 & 0.19 & 23.17 & 22.04 & 22.04 & 0.15 \\
\hline Weight of grains spike (g) & 2.25 & 2.00 & 1.96 & 0.09 & 2.42 & 2.04 & 2.00 & 0.09 \\
\hline 1000-grain weight $(\mathrm{g})$ & 45.21 & 50.62 & 46.33 & 0.54 & 45.54 & 50.58 & 46.58 & 0.29 \\
\hline Biological yield $\left(\mathrm{kg} \mathrm{fed}^{-1}\right)$ & 7359 & 6911 & 6801 & 80.15 & 7362 & 7182 & 6799 & 8.1 \\
\hline Grain yield $\left(\mathrm{kg} \mathrm{fed}^{-1}\right)$ & 1849 & 1657 & 1794 & 3.2 & 1852 & 1660 & 1795 & 3.2 \\
\hline
\end{tabular}

-Effect of different planting methods.

Results in Table (2) indicate that grain yield and its components were affected by different planting methods. Plant height, spike length, No.tillers and spikes $\mathrm{m}^{-2}$, number of spikelets spike ${ }^{-1}$, weight of grains spike $^{-1}$, 1000-grain weight, biological and grain yields fed $^{-1}$ increased significantly by planting methods in the first and second seasons. Rows on beds method increased significantly plant height, spike length, No. tillers and spikes $\mathrm{m}^{-2}$, number of spikelets spike $^{-1}$, weight of grains spike ${ }^{-1}, 1000$-grain weight, biological and grain yields fed $^{-1}$ compared other planting methods in the first and second seasons. Amongst many factors crop production the patter of planting greater significance, as it is not only determined the proper crop stand establishment but also the production of individual plant through balancing the plant to plant competition and facilitating the conversion of light energy to harvest yield of crop. Similar results were reported by Sikander etal, (2003); Sulieman (2010); Abd ElLattief (2011); Dagash etal, (2014); Naresh etal, (2014); Abdul Majeed etal, (2015) and Tadesse etal, (2017).

Table 2.Wheat yield and its components as affected by planting methods in 2014/15 and2015/16 seasons

\begin{tabular}{|c|c|c|c|c|c|c|c|c|c|c|}
\hline \multirow[b]{2}{*}{ Characters } & \multicolumn{4}{|c|}{ 2014/15 season } & \multirow[b]{2}{*}{$\begin{array}{l}\text { L.S.D } \\
\text { at } 5 \%\end{array}$} & \multicolumn{4}{|c|}{ 2015/16 season } & \multirow[b]{2}{*}{$\begin{array}{l}\text { L.S.D } \\
\text { at } 5 \%\end{array}$} \\
\hline & $\begin{array}{l}\text { Prod- } \\
\text { casting }\end{array}$ & Rows & $\begin{array}{l}\text { Hills } \\
\text { on } \\
\text { ridges }\end{array}$ & $\begin{array}{c}\text { Rows } \\
\text { on } \\
\text { beds }\end{array}$ & & $\begin{array}{l}\text { Prod- } \\
\text { casting }\end{array}$ & Rows & $\begin{array}{c}\text { Hills } \\
\text { on } \\
\text { ridges }\end{array}$ & $\begin{array}{c}\text { Rows } \\
\text { on } \\
\text { beds }\end{array}$ & \\
\hline Plant height (cm) & 108.6 & 110.9 & 109.6 & 112.3 & 0.261 & 108.8 & 110.9 & 109.8 & 112.4 & 0.223 \\
\hline Spike length (cm) & 9.39 & 10.28 & 9.83 & 10.33 & 0.223 & 9.50 & 10.33 & 10.06 & 10.33 & 0.126 \\
\hline No. of tillers $\mathrm{m}^{-2}$ & 196.1 & 220.3 & 206.0 & 230.2 & 1.094 & 196.4 & 221.6 & 207.3 & 230.9 & 0.826 \\
\hline No. of spikes $\mathrm{m}^{-2}$ & 193.7 & 217.3 & 203.8 & 227.6 & 1.241 & 194.7 & 218.1 & 205.3 & 228.3 & 0.915 \\
\hline $\begin{array}{l}\text { No. of spikelets } \\
\text { spike }\end{array}$ & 22.17 & 22.16 & 22.44 & 22.72 & 0.208 & 22.28 & 22.72 & 22.50 & 22.94 & 0.151 \\
\hline $\begin{array}{l}\text { Wt. of grains } \\
\text { spike (g) }\end{array}$ & 1.94 & 2.00 & 2.00 & 2.33 & 0.083 & 2.00 & 2.11 & 2.11 & 2.39 & 0.223 \\
\hline $\begin{array}{l}\text { 1000-grain weight } \\
\text { (g) }\end{array}$ & 46.33 & 47.61 & 46.56 & 49.06 & 0.777 & 46.39 & 47.67 & 46.94 & 49.28 & 0.722 \\
\hline $\begin{array}{l}\text { Biological yield } \\
\left(\mathrm{kg} \mathrm{fed}^{-1}\right)\end{array}$ & 6725 & 7096 & 7089 & 7184 & 5.202 & 7080 & 7099 & 7090 & 7187 & 4.882 \\
\hline $\begin{array}{l}\text { Grain yield (kg } \\
\text { fed }^{-1} \text { ) }\end{array}$ & 1758 & 1765 & 1766 & 1777 & 4.287 & 1759 & 1767 & 1770 & 1778 & 3.186 \\
\hline
\end{tabular}

-Effect of nitrogen application methods.

Results in Table (3) show in general that nearly all wheat characters were significantly affected by $\mathrm{N}$ application methods (prodcasting and drilling) in both seasons except No. spikelets spike ${ }^{-1}$ in both seasons, weight of grains spike and grain yield $\mathrm{fed}^{-1}$ in the first season, spike length and 1000-grain weight in the second one. However, prodcasting $\mathrm{N}$ application method increased significantly in plant height, No. tillers $\mathrm{m}^{-2}$, No. spikes $\mathrm{m}^{-2}$ and biological yield $\mathrm{fed}^{-1}$ in the two seasons, spike length and 1000grain weight in the first season, weight of grains spike and grain yield fed ${ }^{-1}$ in the second season.
The effectiveness of these inhibitors in increasing yield and improving yield components is affected by several soil and environmental factors. Moreover, the optimal application method and timing of nitrogen fertilizer was not well defined for farmers. Therefore, we evaluated the effect of inhibitors, timing and method of nitrogen fertilizer application on grain yield, yield components and residual soil mineral nitrogen content. Nitrogen fertilizer application caused a significant increase in grain yield, yield components of wheat in both seasons. 
Table 3. Wheat yield and its components as affected by nitrogen applying methods in 2014/15 and 2015/16 seasons

\begin{tabular}{|c|c|c|c|c|c|c|}
\hline \multirow{2}{*}{ Characters } & \multicolumn{2}{|c|}{$2014 / 15$ season } & \multirow{2}{*}{$\begin{array}{l}\text { Signif- } \\
\text { cance }\end{array}$} & \multicolumn{2}{|c|}{$2015 / 16$ season } & \multirow{2}{*}{$\begin{array}{c}\text { Signifi- } \\
\text { cance }\end{array}$} \\
\hline & Prodcasting & Drilling & & Prodcasting & Drilling & \\
\hline Plant height (cm) & 110.5 & 110.2 & $* *$ & 110.6 & 110.3 & $* *$ \\
\hline Spike length (cm) & 10.03 & 9.89 & $* *$ & 10.11 & 10.00 & NS \\
\hline No. of tillers $\mathbf{m}^{-2}$ & 215.9 & 210.4 & $* *$ & 216.6 & 211.5 & $* *$ \\
\hline No. of spikes $\mathbf{m}^{-2}$ & 213.0 & 208.2 & $* *$ & 214.1 & 209.1 & $* *$ \\
\hline No. of spikelets spike & 22.56 & 22.42 & NS & 22.64 & 22.58 & NS \\
\hline Wt. of grains spike (g) & 2.083 & 2.056 & NS & 2.194 & 2.111 & $*$ \\
\hline 1000-grain weight (g) & 47.58 & 47.19 & $* *$ & 47.72 & 47.42 & NS \\
\hline Biological yield $\left(\mathrm{kg} \mathrm{fed}^{-1}\right)$ & 7109 & 6938 & $* *$ & 7119 & 7109 & $* *$ \\
\hline Grain yield $\left(\mathrm{kg} \mathrm{fed}^{-1}\right)$ & 1767 & 1766 & NS & 1770 & 1767 & $*$ \\
\hline
\end{tabular}

$*$ and ** indicates significant at $P<0.05$ and 0.01 ,

Similar results were also obtained by TeixeiraFilho et al (2007) and Mohammed et al, (2013)

\section{D- Effect of the interactions:}

The significant interactions among wheat varieties, planting methods and nitrogen applying methods on the studied traits are shown in Tables $(4 \mathrm{a}, 4 \mathrm{~b}, 4 \mathrm{c}$ and 5$)$

The combination of wheat varieties with planting methods indicated that the highest values were obtained by Misr 2 variety + rows on beds method for plant height, No. tillers and spikes $\mathrm{m}^{-2}$, No.spikelets spike ${ }^{-1}$, weight of grains spike $^{-1}$, biological and grain yields $\mathrm{fed}^{-1}$, whereas by Sakha 94 variety + rows on beds method gave the heaviest value for 1000-grain weight in the first and second seasons (Table 4A).

On the other hand, the lowest values in plant height, No. tillers and spikes $\mathrm{m}^{-2}$, No. spikelets spike${ }^{1}$ and grain yield $\mathrm{fed}^{-1}$ were obtained by Sakha 94 variety + prodcasting planting method, in 1000-grain weight was obtained by Misr 2 variety + prodcasting planting method and in biological yield fed $^{-1}$ was obtained by Sids 12 variety+ prodcasting planting method in the first and second seasons.

The combination of wheat varieties with $\mathrm{N}$ application methods showed that the highest values were obtained by Misr 2 variety + prodcasting $\mathrm{N}$ application method for plant height, No. tillers and spikes $\mathrm{m}^{-2}$, No.spikelets spike ${ }^{-1}$ and biological yields fed $^{-1}$, while by Sakha 94 variety + prodcasting $N$ application method gave the heaviest values for 1000-grain weight in both seasons are shown Table
$\mathrm{NS}=$ No significance

(4b). On the other hand, Sakha 94 variety + drilling $\mathrm{N}$ application method gave the lowest values in plant height, No.tillers and spikes $\mathrm{m}^{-2}$, No.spikelets spike ${ }^{-1}$ and biological yield $\mathrm{fed}^{-1}$ and Misr 2 variety + drilling $\mathrm{N}$ application method gave the lowest value for 1000-grain weight in both seasons.

The data presented in Table (4c) demonstrated that maximum values were gained in plant height, No. of tillers and spikes $\mathrm{m}^{-2}$, No. of spikelets spike ${ }^{-1}$, biological and grain yields $\mathrm{fed}^{-1}$ by rows on beds method + prodcasting $\mathrm{N}$ application method, whereas the lowest values in these traits were exhibited by prodcasting planting method + drilling $\mathrm{N}$ application method in the first and second seasons.

Misr 2 variety + rows on beds method + prodcasting $\mathrm{N}$ application method gave the highest values of plant height, No tillers and spikes $\mathrm{m}^{-2}$, No.spikelets spike-1, weight of grains spike ${ }^{-1}$, biological and grain yields $\mathrm{fed}^{-1}$, whereas by Sakha 94 variety + rows on beds method+ prodcasting $\mathrm{N}$ application method gave the heaviest value for 1000-grain weight in the first and second seasons (Table 5). On the other hand, Sakha 94 variety + prodcasting planting method + drilling $\mathrm{N}$ application method gave the lowest values in plant height, No. tillers and spikes $\mathrm{m}^{-2}$, No.spikelets spike ${ }^{-1}$ and grain yield $\mathrm{fed}^{-1}$, Misr 2 variety + prodcasting planting method + drilling $\mathrm{N}$ application method gave the lowest value in 1000-grain weight and the biological yield fed $^{-1}$ was exhibited by Sids 12 variety+ prodcasting planting method + drilling $\mathrm{N}$ application method in both seasons. 
Table 4 A. Effect of the interaction between wheat varieties and planting methods on yield and yield components in 2014/15 (First) and2015/16 (Second) seasons

\begin{tabular}{|c|c|c|c|c|c|c|c|c|c|c|c|c|c|c|}
\hline \multirow[b]{2}{*}{ Characters } & \multirow{2}{*}{$\begin{array}{l}\text { Varieties } \\
\text { Planting } \\
\text { methods } \\
\end{array}$} & \multicolumn{4}{|c|}{ Misr 2} & \multicolumn{4}{|c|}{ Sakha 94} & \multicolumn{4}{|c|}{ Sids 12} & \multirow{2}{*}{$\begin{array}{l}\text { LSD } \\
\text { at } 5 \%\end{array}$} \\
\hline & & $\mathbf{P}$ & $\mathbf{R}$ & HR & $\mathbf{R B}$ & $\mathbf{P}$ & $\mathbf{R}$ & HR & $\mathbf{R B}$ & $\mathbf{P}$ & $\mathbf{R}$ & HR & RB & \\
\hline \multirow{2}{*}{ Plant height (cm) } & First & 111.8 & 115.0 & 112.8 & 116.8 & 105.2 & 107.5 & 106.5 & 108.7 & 108.8 & 110.2 & 109.5 & 111.3 & 0.18 \\
\hline & Second & 112.0 & 115.0 & 112.8 & 117.0 & 105.2 & 107.7 & 106.5 & 108.7 & 109.2 & 110.2 & 110.0 & 111.5 & 0.387 \\
\hline \multirow{2}{*}{$\begin{array}{l}\text { No. of tillers } \\
\mathbf{m}^{-2}\end{array}$} & First & 229.2 & 257.7 & 237.0 & 268.2 & 172.5 & 189.0 & 181.0 & 194.8 & 186.5 & 214.3 & 200.0 & 227.7 & 1.893 \\
\hline & Second & 229.2 & 258.7 & 238.0 & 269.5 & 173.0 & 190.2 & 182.0 & 195.7 & 187.0 & 215.8 & 201.8 & 227.7 & 1.431 \\
\hline \multirow{2}{*}{ No. of spikes $\mathbf{m}^{-2}$} & First & 226.2 & 256.5 & 234.8 & 264.3 & 171.5 & 185.2 & 179.3 & 192.2 & 183.3 & 210.3 & 197.3 & 226.3 & 2.149 \\
\hline & Second & 226.5 & 257.2 & 235.0 & 265.7 & 171.7 & 185.5 & 181.7 & 192.8 & 185.8 & 211.7 & 199.3 & 226.3 & 1.585 \\
\hline \multirow{2}{*}{ No. of spikelets spike ${ }^{-1}$} & First & 22.67 & 23.00 & 23.00 & 23.33 & 21.83 & 22.00 & 22.00 & 21.83 & 22.00 & 22.83 & 22.33 & 23.00 & 0.359 \\
\hline & Second & 22.83 & 23.00 & 23.00 & 23.83 & 22.00 & 22.17 & 22.00 & 22.00 & 22.00 & 23.00 & 22.50 & 23.00 & 0.261 \\
\hline \multirow{2}{*}{ Wt. of grains spike (g) } & First & 2.00 & 2.00 & 2.00 & 3.00 & 2.00 & 2.00 & 2.00 & 2.00 & 1.83 & 2.00 & 2.00 & 2.00 & 0.143 \\
\hline & Second & 2.00 & 2.33 & 2.33 & 3.00 & 2.00 & 2.00 & 2.17 & 2.00 & 2.00 & 2.00 & 2.00 & 2.00 & 0.387 \\
\hline \multirow{2}{*}{ 1000-grain weight (g) } & First & 44.83 & 45.33 & 43.83 & 46.83 & 47.67 & 51.50 & 50.50 & 52.83 & 46.50 & 46.00 & 45.33 & 47.50 & 1.45 \\
\hline & Second & 45.50 & 45.50 & 44.33 & 46.83 & 47.67 & 51.17 & 50.50 & 53.00 & 46.00 & 46.33 & 46.00 & 48.00 & NS \\
\hline \multirow{2}{*}{ Biological yield $\left(\mathrm{kg} \mathrm{fed}^{-1}\right)$} & First & 7333 & 7404 & 7370 & 7328 & 6084 & 7229 & 7165 & 7166 & 6757 & 6919 & 6755 & 6773 & NS \\
\hline & Second & 7330 & 7411 & 7370 & 7334 & 7160 & 7228 & 7174 & 7166 & 6750 & 6920 & 6753 & 6771 & 8.456 \\
\hline \multirow{2}{*}{$\begin{array}{l}\text { Grain yield } \\
\left(\mathrm{kg} \mathrm{fed}^{-1}\right)\end{array}$} & First & 1847 & 1844 & 1846 & 1860 & 1648 & 1652 & 1659 & 1667 & 1779 & 1799 & 1794 & 1804 & 7.426 \\
\hline & Second & 1850 & 1846 & 1850 & 1861 & 1650 & 1658 & 1664 & 1668 & 1779 & 1798 & 1797 & 1805 & 5.52 \\
\hline $\begin{array}{l}\mathbf{P}=\text { Prodcasting method } \quad \mathrm{R}=1 \\
\end{array}$ & $\begin{array}{ll}\text { ws method } \quad H R=F \\
\end{array}$ & ridges meth & $\mathrm{od} \quad \mathrm{RB}=1$ & ws on bed & nethod & $\mathrm{NS}=\mathrm{No} \mathrm{si}$ & ificance & & & & & & & \\
\hline
\end{tabular}


Table 4 B. Effect of the interaction between wheat varieties and nitrogen applying methods on yield and yield components in 2014/15 (First) and 2015/16 (Second) seasons

\begin{tabular}{|c|c|c|c|c|c|c|c|c|}
\hline \multirow[t]{2}{*}{ Characters } & \multirow{2}{*}{$\begin{array}{c}\text { Varieties } \\
\text { N. applying methods }\end{array}$} & \multicolumn{2}{|c|}{ Misr 2} & \multicolumn{2}{|c|}{ Sakha 94} & \multicolumn{2}{|c|}{ Sids 12} & \multirow{2}{*}{$\begin{array}{r}\text { LSD } \\
\text { at } 5 \%\end{array}$} \\
\hline & & $\mathbf{P}$ & D & $\mathbf{P}$ & D & $\mathbf{P}$ & D & \\
\hline \multirow[b]{2}{*}{ Plant height $(\mathrm{cm})$} & First & 114.2 & 114.1 & 107.2 & 106.8 & 110.2 & 109.7 & NS \\
\hline & Second & 114.2 & 114.3 & 107.3 & 106.7 & 110.4 & 110.0 & 0.243 \\
\hline \multirow{2}{*}{ No. of tillers $\mathbf{m}^{-2}$} & First & 250.58 & 245.42 & 185.92 & 182.75 & 211.25 & 203.00 & 1.805 \\
\hline & Second & 251.42 & 246.25 & 186.25 & 184.17 & 212.08 & 204.08 & 1.088 \\
\hline \multirow{2}{*}{ No. of spikes $\mathrm{m}^{-2}$} & First & 248.0 & 242.9 & 183.3 & 180.7 & 207.7 & 201.0 & 0.855 \\
\hline & Second & 248.5 & 243.7 & 184.2 & 181.6 & 209.5 & 202.1 & 1.253 \\
\hline \multirow{2}{*}{ No. of spikelets spike $e^{-1}$} & First & 23.08 & 22.92 & 22.92 & 22.92 & 22.67 & 22.42 & NS \\
\hline & Second & 23.17 & 23.17 & 22.00 & 22.08 & 22.75 & 22.50 & 0.172 \\
\hline \multirow{2}{*}{ 1000-grain weight(g) } & First & 45.42 & 45.00 & 51.08 & 50.17 & 46.25 & 46.42 & 0.421 \\
\hline & Second & 45.83 & 45.25 & 51.08 & 50.08 & 46.25 & 46.92 & 0.795 \\
\hline \multirow{2}{*}{$\begin{array}{l}\text { Biological yield } \\
\left(\text { ton fed }^{-1}\right)\end{array}$} & First & 7366 & 7352 & 6640 & 7182 & 6809 & 6794 & NS \\
\hline & Second & 7370 & 7353 & 7179 & 7184 & 6807 & 6790 & $\mathbf{5 . 5 7 3}$ \\
\hline
\end{tabular}

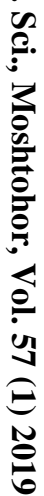

NS=No significance 
Table 4C. Effect of the interaction between planting methods and nitrogen applying methods on yield and yield components in 2014/15 (First) and 2015/16 (Second) seasons

\begin{tabular}{|c|c|c|c|c|c|c|c|c|c|c|}
\hline \multirow{2}{*}{ Characters } & \multirow{2}{*}{$\begin{array}{c}\text { Planting methods } \\
\begin{array}{c}\text { N. applying } \\
\text { methods }\end{array}\end{array}$} & \multicolumn{2}{|c|}{ Prodcasting method } & \multicolumn{2}{|c|}{$\begin{array}{c}\text { Rows } \\
\text { method }\end{array}$} & \multicolumn{2}{|c|}{ Hill on ridges method } & \multicolumn{2}{|c|}{ Rows on bed method } & \multirow{2}{*}{$\begin{array}{c}\text { LSD } \\
\text { at } 5 \%\end{array}$} \\
\hline & & $\mathbf{P}$ & D & $\mathbf{P}$ & D & $\mathbf{P}$ & $\mathbf{D}$ & $\mathbf{P}$ & D & \\
\hline \multirow{2}{*}{ Plant height (cm) } & First & 108.7 & 108.5 & 111.1 & 110.7 & 109.8 & 109.4 & 112.4 & 112.1 & NS \\
\hline & Second & 108.8 & 108.8 & 111.2 & 110.7 & 109.9 & 109.7 & 112.7 & 112.1 & 0.281 \\
\hline \multirow{2}{*}{$\begin{array}{l}\text { No. of tillers } \\
\mathbf{m}^{-2}\end{array}$} & First & 199.8 & 192.3 & 223.0 & 217.7 & 208.9 & 203.1 & 232.0 & 228.4 & NS \\
\hline & Second & 200.1 & 192.7 & 224.1 & 219.0 & 209.8 & 204.8 & 232.3 & 229.6 & 1.256 \\
\hline \multirow{2}{*}{ No. of spikes $\mathbf{m}^{-2}$} & First & 196.1 & 191.2 & 219.3 & 215.3 & 207.5 & 200.1 & 229.0 & 226.2 & 0.987 \\
\hline & Second & 197.3 & 192.0 & 219.8 & 216.4 & 210.0 & 200.7 & 229.2 & 227.3 & 1.446 \\
\hline \multirow{2}{*}{$\begin{array}{l}\text { No. of spikelets } \\
\text { spike }^{-1}\end{array}$} & First & 22.22 & 22.11 & 22.67 & 22.56 & 22.56 & 22.33 & 22.78 & 22.67 & NS \\
\hline & Second & 22.22 & 22.33 & 22.67 & 22.78 & 22.67 & 22.33 & 23.00 & 22.89 & 0.199 \\
\hline \multirow{2}{*}{$\begin{array}{l}\text { Biological yield } \\
\left.\text { (ton fed } \text { fed }^{-1}\right)\end{array}$} & First & 6381 & 7069 & 7097 & 7097 & 7089 & 7089 & 7186 & 7183 & NS \\
\hline & Second & 7096 & 7065 & 7101 & 7097 & 7091 & 7090 & 7187 & 7187 & 6.435 \\
\hline \multirow{2}{*}{$\begin{array}{l}\text { Grain yield } \\
\left.\text { (ton fed }^{-1}\right)\end{array}$} & First & 1657 & 1656 & 1795 & 1793 & 1796 & 1793 & 1850 & 1848 & NS \\
\hline & Second & 1763 & 1756 & 1769 & 1766 & 1773 & 1768 & 1780 & 1775 & 4.376 \\
\hline
\end{tabular}
$\mathrm{P}=$ Prodcasting method $\quad \mathrm{D}=$ drilling method $\mathrm{NS}=$ No significance 
Table 5. Effect of the interaction among wheat varieties, planting methods and nitrogen applying methods on yield and yield components in 2014/15 (F) and 2015/16 (S) seasons

\begin{tabular}{|c|c|c|c|c|c|c|c|c|c|c|c|c|c|c|c|c|}
\hline \multirow{2}{*}{ Varieties } & \multirow{2}{*}{$\mathbf{P M}$} & \multirow{2}{*}{ NAM } & \multicolumn{2}{|c|}{$\begin{array}{l}\text { Plant height } \\
(\mathrm{cm})\end{array}$} & \multicolumn{2}{|c|}{ No. of tillers $\mathbf{~ m}^{-2}$} & \multicolumn{2}{|c|}{ No. of spikes $\mathbf{m}^{-2}$} & \multicolumn{2}{|c|}{$\begin{array}{l}\text { Wt. of grains } \\
\text { spike (g) }\end{array}$} & \multicolumn{2}{|c|}{$\begin{array}{l}\text { 1000-grain } \\
\text { weight (g) }\end{array}$} & \multicolumn{2}{|c|}{$\begin{array}{c}\text { Biological yield } \\
\left.\text { (ton fed }^{-1}\right)\end{array}$} & \multicolumn{2}{|c|}{$\begin{array}{c}\text { Grain yield } \\
\left.\text { (ton fed }^{-1}\right)\end{array}$} \\
\hline & & & $\mathbf{F}$ & $\mathbf{S}$ & $\mathbf{F}$ & $\bar{S}$ & $\bar{F}$ & $\mathbf{S}$ & $\mathbf{F}$ & $\mathbf{S}$ & $\mathbf{F}$ & $\mathbf{S}$ & $\mathbf{F}$ & S & $\mathbf{F}$ & $\mathbf{S}$ \\
\hline \multirow{9}{*}{ Misr 2} & \multirow{2}{*}{$\mathbf{P}$} & $\mathbf{P}$ & 112.0 & 112.0 & 232.3 & 231.3 & 227.7 & 227.3 & 2.00 & 2.00 & 45.33 & 46.00 & 7350 & 7350 & 1848 & 1852 \\
\hline & & D & 111.7 & 112.0 & 226.0 & 227.0 & 224.7 & 225.7 & 2.00 & 2.00 & 44.33 & 45.00 & 7316 & 7311 & 1847 & 1848 \\
\hline & \multirow[t]{2}{*}{$\mathbf{R}$} & $\mathbf{P}$ & 115.0 & 115.0 & 262.0 & 262.7 & 260.0 & 260.3 & 2.00 & 2.33 & 45.33 & 46.00 & 7374 & 7378 & 1858 & 1856 \\
\hline & & D & 115.0 & 115.0 & 253.3 & 254.7 & 253.0 & 254.0 & 2.00 & 2.33 & 45.33 & 45.00 & 7367 & 7363 & 1862 & 1865 \\
\hline & \multirow[t]{2}{*}{ HR } & $\mathbf{P}$ & 113.0 & 112.7 & 240.0 & 241.0 & 239.0 & 240.0 & 2.00 & 2.67 & 44.00 & 44.33 & 7337 & 7343 & 1844 & 1848 \\
\hline & & D & 112.7 & 113.0 & 234.0 & 235.0 & 230.7 & 230.0 & 2.00 & 2.00 & 43.67 & 44.33 & 7319 & 7325 & 1848 & 1852 \\
\hline & \multirow[t]{2}{*}{$\mathbf{R B}$} & $\mathbf{P}$ & 116.7 & 117.0 & 268.0 & 270.7 & 265.3 & 266.3 & 3.00 & 3.00 & 47.00 & 47.00 & 7403 & 7407 & 1844 & 1848 \\
\hline & & D & 117.0 & 117.0 & 268.3 & 268.3 & 263.3 & 265.0 & 3.00 & 3.00 & 46.67 & 46.67 & 7406 & 7416 & 1844 & 1844 \\
\hline & \multirow[t]{2}{*}{$\mathbf{P}$} & $\mathbf{P}$ & 105.0 & 105.0 & 174.3 & 175.0 & 172.3 & 172.0 & 2.00 & 2.00 & 48.00 & 48.00 & 7171 & 7148 & 1645 & 1644 \\
\hline \multirow{5}{*}{ Sakha 94} & & D & 105.3 & 105.3 & 170.7 & 171.0 & 170.7 & 171.3 & 2.00 & 2.00 & 47.33 & 47.33 & 7168 & 7172 & 1652 & 1656 \\
\hline & \multirow[t]{2}{*}{$\mathbf{R}$} & $\mathbf{P}$ & 108.0 & 108.3 & 189.7 & 190.0 & 186.0 & 186.0 & 1.83 & 2.00 & 52.00 & 51.33 & 7234 & 7231 & 1669 & 1667 \\
\hline & & D & 107.0 & 107.0 & 188.3 & 190.3 & 184.3 & 185.0 & 2.00 & 2.00 & 51.00 & 51.00 & 7224 & 7225 & 1666 & 1669 \\
\hline & \multirow[t]{2}{*}{ HR } & $\mathbf{P}$ & 106.7 & 107.0 & 184.0 & 184.0 & 181.0 & 184.0 & 2.00 & 2.00 & 50.67 & 51.00 & 7164 & 7174 & 1659 & 1664 \\
\hline & & D & 106.3 & 106.0 & 178.0 & 180.0 & 177.7 & 179.3 & 2.00 & 2.00 & 50.33 & 50.00 & 7167 & 7174 & 1659 & 1663 \\
\hline \multirow{11}{*}{ Sids 12} & \multirow[t]{2}{*}{ RB } & $\mathbf{P}$ & 109.0 & 109.0 & 195.7 & 196.0 & 194.0 & 195.0 & 2.00 & 2.33 & 53.67 & 54.00 & 7163 & 7165 & 1653 & 1659 \\
\hline & & D & 108.3 & 108.3 & 194.0 & 195.3 & 190.3 & 190.7 & 2.00 & 2.00 & 52.00 & 52.00 & 7169 & 7167 & 1651 & 1658 \\
\hline & \multirow[t]{2}{*}{$\mathbf{P}$} & $\mathbf{P}$ & 109.0 & 109.3 & 192.7 & 194.0 & 188.3 & 192.7 & 2.00 & 2.00 & 47.00 & 46.00 & 6793 & 6789 & 1775 & 1772 \\
\hline & & D & 108.7 & 109.0 & 180.3 & 180.0 & 178.3 & 179.0 & 1.67 & 2.00 & 46.00 & 46.00 & 6721 & 6712 & 1784 & 1785 \\
\hline & \multirow[t]{2}{*}{$\mathbf{R}$} & $\mathbf{P}$ & 110.3 & 110.3 & 217.3 & 219.7 & 212.0 & 213.0 & 2.00 & 2.00 & 46.00 & 47.00 & 6768 & 6766 & 1802 & 1802 \\
\hline & & D & 110.0 & 110.0 & 211.3 & 212.0 & 208.7 & 210.3 & 2.00 & 2.00 & 46.00 & 45.67 & 6779 & 6777 & 1806 & 1807 \\
\hline & \multirow[t]{2}{*}{ HR } & $\mathbf{P}$ & 109.7 & 110.0 & 202.7 & 204.3 & 202.7 & 206.0 & 2.00 & 2.00 & 45.00 & 45.00 & 6754 & 6752 & 1803 & 1807 \\
\hline & & D & 109.3 & 110.0 & 197.3 & 199.3 & 192.0 & 192.7 & 2.00 & 2.00 & 45.67 & 47.00 & 6756 & 6754 & 1785 & 1788 \\
\hline & \multirow[t]{2}{*}{ RB } & $\mathbf{P}$ & 111.7 & 112.0 & 232.3 & 230.3 & 227.7 & 226.3 & 2.00 & 2.00 & 47.00 & 47.00 & 6920 & 6921 & 1792 & 1790 \\
\hline & & D & 111.0 & 111.0 & 223.0 & 225.0 & 225.0 & 226.3 & 2.00 & 2.00 & 48.00 & 49.00 & 6919 & 6919 & 1806 & 1806 \\
\hline & at $5 \%$ & & NS & 0.487 & 3.609 & 2.177 & 1.709 & 2.505 & NS & 0.281 & 0.843 & NS & NS & 11.146 & 9.748 & 7.579 \\
\hline
\end{tabular}

PM=Planting methods NAM=N. applying methods $\mathrm{P}=$ Prodcasting method $\mathrm{D}=$ drilling method R=Rows method HR=Hill on ridges method RB= Rows on bed method 
It could be concluded that under the conditions of the experiment planting Misr 2 variety by rows on bed method with prodcasting $\mathrm{N}$ application method is recommended.

\section{References}

Abd El-Hameed, I. A. (2002). Effect of some agromomic practices on wheat. Ph. D. Thesis, Fac. Agric., Zagazig Univ., Egypt.

Abd El-Lattief, E. A. (2011). Bread wheat (Triticum aestivum L.) productivity and profitability as affected by method of sowing and seeding rate under Qena environment. Asian J. of Crop Science 3 (4): 188-196.

Abd El-Nour, Nadya, A. R. and Hayam S. A. Fateh (2011). Influence of sowing date and nitrogen fertilization on yield and its components in some bread wheat genotypes. Egypt. J. Agric. Res., 89(4):1413-1433.

Abdul Majeed, A. Muhmood, A. Niaz, S. Javid, Z. A. Ahmad, S. S. H. Shah and A. H. Shah (2015). Bed planting of wheat (Triticum aestivum L.) improves nitrogen use efficiency and grain yield compared to flat planting. The Crop J. 3: $118-124$.

Abu-Grab, O. S., A. M. Mousa and G. A. ElShaarawy (2006). Photosynthetic and N-use efficiencies for some wheat cultivars in relation to planting density and nitrogen fertilization level. Egypt. J. Appl. Sci., 21(2B): 475-492.

Abo-Remaila, S.I. and M.M. Abou El Enim (2017). Effect of some phosphorus fertilizer packages on growth and yield of some Egyptian wheat varieties. Advances in Crop Science and Technology(Adv Crop Sci Tech), 5 (2):2-7.

Ali, G. A., O. E. Zeiton, A. H. Bassiouny and A. R. Y. A. El-Banna (2004). Productivity of wheat cultivars grown at El-Khattara and El-Arish under different levels of planting densities and $\mathrm{N}$ fertilization. Zagazig J. Agric. Res., 31(4A): 1225-1256.

Ashmawy, F., M. S. El-Habal, H. S. Saoudy and ImanKh. Abbas (2010). The relative contribution of yield components to grain yield of some wheat cultivars grown under different nitrogen fertilizer levels. Egypt. J. Agric. Res., 88(1):225-239.

Dagash Y.M.I.; I.M.M. Syed Ahmed and N.A. Khalil (2014). Effect of nitrogen fertilization, sowing methods and sowing dates on yield and yield attributes of wheat (Triticum aestivum L). Universal Journal of Plant Science 2(6): 108-113.

El-Hawary, M. A. (2000). Evaluation of some wheat varieties under water deficit conditions. Zagazig J. Agric. Res., 27(4): 819-830.

Hassan, Manal A. (2008). Effect of seeding rate and row spacing on productivity and resistance to powdery mildew of two bread wheat cultivars. Egypt. J. Appl. Sci., 23(10A): 169-182.
Mehasen, S. A. S. (1999). Response of some wheat varieties to agrispon foliar application and nitrogen rates. Annals of Agric. Sci., Moshtohor, 37(2): $853-864$.

Mehasen, S. A. S and N. A. Mohamed (2005). Multivariate and response curve analyses for yield and its attributes in some wheat cultivars under nitrogen fertilization levels. Egypt. J. Appl. Sci., 20(2): 93-109.

Mehasen, S. A. S., El-Gizawy, N. Kh., Sharoba, A. M., Soliman, S. A. and Khalil, T. R. M. 2014. Yield and chemical composition of bread wheat cultivars as affected by some skipping irrigation. Minufiya J. Agric. Res. 39(3):1009-1018.

Mehasen, S. A. S., M. A. Ahmed and M. A. M. Morsy (2009). Evaluation of some wheat genotypes under different seeding rates.Annals of Agric. Sci., Moshtohor, 47 (3):167-174.

Mehasen, S. A. S., M. A. Ahmed and S. Sh. Abdullah (2013). Improving productivity of bread wheat genotypes by using of some growth promoters. Egypt. J. Plant Breed., 17(2):4-15.

Mehasen, S.A.S.; Shimaa A. Badawy and S. Sh. Abdullah (2015). Influence of bio and mineral nitrogen fertilizers on productivity of some bread wheat varieties. J. of Food, Agriculture \& Environment Vol.13 (2): 162-167.

Michigan State University (1983). MSTAT-C: Micro- computer Statistical Program, Version 2.0. Michigan State University, East Lansing.

Mohammed, Y. A.; T. Jensen; J. Heser and C. Chen (2013). Inhibitors, method and time of nitrogen application for improved winter wheat production in central Montana. Western Nutrient Management Conference, 10:108-113.

Naresh R. K.; S. S.Tomar; P. shottam; S. P. Singh; D. Kumar, B. Pratap; V. Kumar and A. H. Nanher (2014). Testing and evaluation of planting methods on wheat grain yield and yield contributing parameters in irrigated agroecosystem of western Uttar Pradesh, India. Afr. J. Agric. Res., 9(1): 176-182.

Sikander, K.T.; I. Hussain; M. Sohail; N. S. Kissana and S. G. Abbas (2003). Effect of different planting methods on yield and yield components of wheat. Asian J. Plant Science 2(10): 811-813.

Snedecor, G. W. and W. G. Cochran (1980). Statistical Methods, $7^{\text {th }}$ Ed., Iowa State Univ. Press, Ames, Iowa, USA.

Tadesse, A.; T. Yoseph; M. Mitiku (2017). Effect of sowing methods and seed rate on yield of bread wheat (Triticum aestivum L.) at South Ari District, South Omo Zone, Snnpr, Ethiopia. International J. of Research-Granthaalayah, 5(6):175-180.

Teixeira-Filho, M.C., M.S. Buzetti, R.C.F. Alvarez, J.G. Freitas, O. Arfe and M.E. Sá (2007). Resposta de cultivares de trigo irrigadoporaspersãoao nitrogênioe mcobertur 
anaregião do Cerrado. Acta Scientiarum-Agron., 29: 421-425.

Omar, A. E. A. (2007). Productivity of some wheat cultivars as affected by sowing date and seeding rate. Egypt. J. Appl. Sci., 22(3): 103-116.

Ullah, G. , E. A. Khan, I. U. Awan, M. A. K. A. Khakwani, M. S. Baloch, Q.U. Khan, M. S. Jilani, K. Wasim, S. Javeria and G. Jilani (2013). Wheat Response to Application Methods and Levels of Nitrogen Fertilizer: I. Phenology,
Growth Indices and Protein Content. Pak. J. Nutr., 12 (4):365-370.

Zenhom, M. F. T; G. Y. Hammam and S. A. S. Mehasen (2018). Wheat lodging and yield in response to cultivars and foliar application of paclobutrazol. 4th International Conference on Biotechnology Applications in Agriculture (ICBAA), 4-7 April 2018, Hurghada, Egypt Invited Papers, 639-644

\section{تأثير طرق الزراعة وإضافة النيتروجين علي المحصول ومكوناتة لبعض اصناف القمح}

صديق عبد العزيز صديق محيسن ، ناصر خميس بركات الجيزاوي ، محمد السيد رياض جمعة و عائشة محمد عبدالرحمن حلاوة ، قسم المحاصيل - كلية الزراعة بمشتهر - جامعة بنها

اجريت تجربتان حقليتان بمركز البحوث والتجارب بكلبة الزراعة بمشتهر - جامعة بنها خلال موسمى 2015/2014 و 2016/2015 م لدراسة

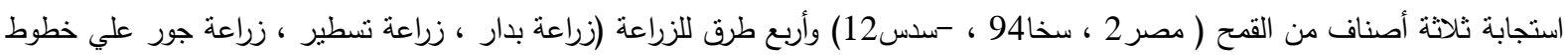
، زراعة تسطبر علي مصاطب) وطربقتين لإضافة السماد الأزوتي (بدار ، سرسبة) علي المحصول وبعض مكوناتة.

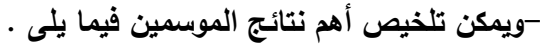
سجل صنف القمح مصر2 أعلى منوسطات لكل من طول النبات ، طول السنبلة ،عدد الأشطاء والسنابل للمنز المربع ، عدد سنيبلات السنبلة ، وزن حبوب السنبلة ، المحصول البيولوجى و محصول الحبوب للفدان. بينما أعطي صنف سخا 94 أعلي منوسط لوزن الـ 1000 حبة مقارنه بالأصناف الأخرى لكلا الموسمين.

ازداد كل من طول النبات ، طول السنبلة ،عدد الأشطاء والسنابل للمتر المربع ، عدد سنيبلات السنبلة ، وزن حبوب السنبلة ، وزن الـ الـ

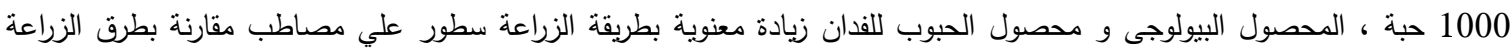
الأخري في كل من موسمي الزراعة الأول والثاني.

أعطت إضافة السماد النيتروجيني بالطريقة البدار إلى زيادة معنوية فى كل من طول النبات ، طول السنبلة ،عدد الأشطاء والسنابل للمنز المربع ، عدد سنيبلات السنبلة ، وزن حبوب السنبلة ، وزن الـ 1000 حبة ، الدحصول البيولوجى و محصول الحبوب للفدان لكلا موسمي فئي الزراعة.

تأثز معنويا كل من طول النبات ، عدد الأشطاء والسنابل للمتر المربع ، عدد سنيبلات السنبلة ، وزن حبوب السنبلة ، وزن الـ 1000 حبة ، المحصول البيولوجى و محصول الحبوب للفدان بالتفاعل بين الأصناف و طرق الزراعة ماعدا وزن الـ 1000 حبة في الموسم الثاني فقط. بينما تأثر معنويا كل من طول النبات ، عدد الأشطاء والسنابل للمتر المربع ، عدد سنيبلات السنبلة ، وزن الـ 1000 حبة والمحصول البيولوجى للفدان بالتفاعل بين الأصناف وطرق إضافة السماد النيتروجين ماعدا طول النبات وعدد سنيبلات السنبلة في الموسم الأول فقط. وكذلك أثر التفاعل بين طرق الزراعة وطرق إضافة السماد النيتروجين تأثير معنويا علي كل من عدد السنابل للمنر المربع والمحصول

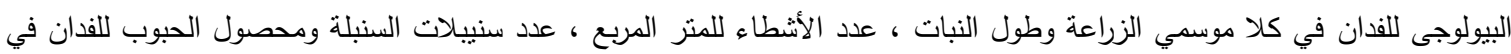
الموسم الثاني فقط. أثز التفاعل بين الأصناف وطرق الزراعة وطرق إضافة السماد النيتروجين تأثثرا معنويا علي كل من عدد الأشطاء والسنابل للمتز المربع ، المحصول البيولوجى و محصول الحبوب للفدان في كل من موسمي الزراعة بينما نأثير معنويا وزن الـ 1000 حبة في الموسم الأول فقط و

$$
\text { كل من طول النبات و وزن حبوب السنبلة في الموسم الثاني فقط. }
$$

توصي هذه الدراسة تحت ظروف تلك التجربة بزراعة صنف القمح مصر 2 بطريقة التسطير علي مصاطب وإضافة السماد النيتروجين نثرا. 